\title{
Reasoning About Solutions in Linear Algebra: the Case of Abraham and the Invertible Matrix Theorem
}

\author{
Megan Wawro ${ }^{1}$
}

Published online: 30 September 2015

(C) Springer International Publishing Switzerland 2015

\begin{abstract}
A rich understanding of key ideas in linear algebra is fundamental to student success in undergraduate mathematics. Many of these fundamental concepts are connected through the notion of equivalence in the Invertible Matrix Theorem (IMT). The focus of this paper is the ways in which one student, Abraham, reasoned about solutions to $A \boldsymbol{x}=0$ and $A \boldsymbol{x}=\boldsymbol{b}$ to draw connections between other concept statements within the IMT. Data sources were video and transcripts from whole class discussion, small group work, and individual interviews. The overarching analytical structure was influenced by a framework of genetic analysis (Saxe, Journal of the Learning Sciences, 11, 275-300, 2002), and Toulmin's Model of Argumentation (1969) was employed to analyze the structure of arguments both in isolation (microgenesis) and over time (ontogenesis). This case study, rather than focusing on student difficulties in undergraduate mathematics, serves as a compelling example of the productive and powerful reasoning that is possible as students make sense of complex mathematics. The results present an ontogenetic analysis of Abraham's use of solutions to reason about how span and linear independence of a set of vectors are related, as well microgenetic analyses of various examples of reasoning about solutions to justify connections between other concepts within the IMT.
\end{abstract}

Keywords Linear algebra $\cdot$ Solutions $\cdot$ Student reasoning $\cdot$ Genetic analysis

Consider the equations $A \boldsymbol{x}=\boldsymbol{b}$ and $A \boldsymbol{x}=0$, where $A$ is an $m \times n$ matrix, $\boldsymbol{x}$ is a vector in $\mathbb{R}^{n}, \boldsymbol{b}$ is a vector in $\mathbb{R}^{\boldsymbol{m}}$, and 0 is the zero vector in $\mathbb{R}^{\boldsymbol{m}}$. How might a student think about these equations? How might a student reason about how solutions to these two matrix equations are related or affect each other? How might knowledge about the existence or uniqueness of solutions to these equations provide insight into other desired

Megan Wawro

mwawro@vt.edu

1 Department of Mathematics (0123), Virginia Tech, Mcbryde Hall 438, 225 Stanger St., Blacksburg, VA 24061-1026, USA 
information in linear algebra, such as the span or linear independence of the column vectors of $A$ ? The fundamental concepts these questions address, for the case in which $A$ is an $n \times n$ matrix, are among the very ones connected through the notion of equivalence in what is known as the Invertible Matrix Theorem (IMT). The focus of this paper is the ways in which one student, Abraham, reasoned about solutions to $A \boldsymbol{x}=$ 0 and $A \boldsymbol{x}=\boldsymbol{b}$ to draw connections between other concept statements within the IMT.

The IMT (see Fig. 1) is a core theorem in linear algebra that connects many fundamental concepts through the notion of equivalence. For the introductory linear algebra classroom community that was the focus of this study, what became known as the IMT developed in an emergent fashion. That is, the various equivalencies took form and developed meaning over time as students came to reason about the ways in which key ideas involved were connected. As the semester progressed, the class investigated the individual concepts within the IMT and related them to one another; the resulting set of relations was the list of 16 equivalent statements (when $A$ is $n \times n$ ) listed in Fig. 1. Because the IMT weaves together many of the central ideas in an introductory course in linear algebra, no one single class session could encompass all the equivalencies that constitute the IMT. Rather, new equivalencies were added to the theorem as the semester progressed. Thus, students' understanding of the theorem changed in two dimensions over the semester: (a) knowledge of the theorem changed as aspects of it were developed and layered on over the course of the semester, and (b) understanding of previously known aspects of the theorem (presumably) changed as the semester progressed.

This paper draws from a larger study that documented the development of mathematical meaning related to the IMT for both a classroom community and an individual student, Abraham, over time (Wawro 2011). The focus of this paper is Abraham's ways of reasoning regarding a portion of the IMT. In particular, it documents the ways in which Abraham reasoned about solutions to $A \boldsymbol{x}=0$ and $A \boldsymbol{x}=\boldsymbol{b}$ to draw connections between concept statements within the IMT. Abraham's reliance on reasoning about the

\section{The Invertible Matrix Theorem}

Let $A$ be an $n \times n$ matrix. The following are equivalent:

1. The columns of $A$ span $\mathbb{R}^{n}$.

2. The matrix $A$ has $n$ pivots.

3. For every $\mathbf{b}$ in $\mathbb{R}^{n}$, there is a solution $\mathbf{x}$ to $A \mathbf{x}=\mathbf{b}$.

4. For every $\mathbf{b}$ in $\mathbb{R}^{n}$, there is a way to write $\mathbf{b}$ as a linear combination of the columns of $A$.

5. $A$ is row equivalent to the $n \times n$ identity matrix.

6. The columns of $A$ form a linearly independent set.

7. The only solution to $A \mathbf{x}=\mathbf{0}$ is the trivial solution.

8. $A$ is invertible.

9. There exists an $n \times n$ matrix $C$ such that $C A=I$.

10. There exists an $n \times n$ matrix $D$ such that $A D=I$.

11. The transformation $\mathbf{x} \rightarrow A \mathbf{x}$ is one-to-one.

12. The transformation $\mathbf{x} \rightarrow A \mathbf{x}$ maps $\mathbb{R}^{n}$ onto $\mathbb{R}^{n}$.

13. $\operatorname{Col} A=\mathbb{R}^{n}$.

14. $\operatorname{Nul} A=\{\mathbf{0}\}$.

15. $\operatorname{Det} A \neq 0$.

16. The matrix $A$ does not have the number zero as an eigenvalue.

Fig. 1 The invertible matrix theorem 
existence and uniqueness of solutions to $A \boldsymbol{x}=0$ and $A \boldsymbol{x}=\boldsymbol{b}$ was prominent throughout the semester. He challenged himself to understand connections between these two matrix equations and their solutions, and this served a key role in helping him develop a way of reasoning about the equivalence of "the columns of $A$ span $\mathbb{R}^{n}$ " and "the columns of $A$ are linearly independent" for an $n \times n$ matrix $A$. Furthermore, Abraham used the notion of solutions to reason about connections between nearly all of the other statements in the IMT. These aspects of his argumentation are in contrast to the argumentation patterns with the whole class discussion at the collective level, within which reasoning about solutions did not surface as central. It is because of these reasons that this paper focuses on Abraham's powerful reasoning about solutions to $A \boldsymbol{x}=0$ and $A \boldsymbol{x}=\boldsymbol{b}$ to draw connections between other concept statements within the IMT.

Investigating the ways of reasoning of one individual student provides a solid foundation for understanding the possible conceptual milestones, blocking points, and potentially helpful and inhibitive ways of reasoning about the concept statements (and their equivalence) within the IMT of other linear algebra students. In other words, what is learned about Abraham's ways of reasoning - the concept statements that were most central to his reasoning, the structure of his argumentation, specific interpretations of concept statements that were particularly salient for him, and the like-serve a conjectural role regarding what may be true for other individuals' ways of reasoning. This case study, rather than focusing on student difficulties in undergraduate mathematics, serves as a compelling example of the productive and powerful reasoning that is possible as students make sense of complex mathematics.

\section{Literature and Significance}

Linear algebra is one of the most useful fields of mathematical study because of its unifying power in the discipline as well as its applicability to areas outside of pure mathematics (Dorier 1995; Strang 1988). According to Harel (1989), linear algebra is an important subject matter at the college level in that it can (a) be applied to many different content areas, such as engineering and statistics, because of its power to model various situations; and (b) be studied in its own right as "a mathematical abstraction which rests upon the pivotal ideas of the postulational approach and proof" (p. 139). Thus, when considering the mathematical development of undergraduate students, a first course in linear algebra plays an important, transitional role. In many universities, a first course in linear algebra follows immediately after a calculus series and often prior to an introduction to proof course. According to Carlson (1993), the majority of students' mathematical experiences up to that point in their education may have been primarily computational in nature. The content of linear algebra, however, can be highly abstract and formal, and this shift in the nature of the mathematical content can be rather difficult for students to handle smoothly. Carlson (1993) posits that concepts are often taught without substantial connection to students' previously learned mathematical ideas, as well as without examples or applications. Thus, students struggle with connecting familiar concepts to prematurely formalized, unfamiliar ones. Indeed, Robert and Robinet (1989) showed that prevalent student criticisms of linear algebra relate to its "use of formalism, the overwhelming amount of new definitions 
and the lack of connection with what they already know in mathematics" (as cited in Dorier et al. 2000, p. 86).

As the quote indicates, the mathematical activities central to linear algebra frequently involve mathematical ideas with which students may have little experience, such as span or linear transformation. How do students understand these ideas and reason about how they are related? Within mathematics education research, there is considerable precedence in characterizing student understanding, in part, as connections between concepts (e.g., NCTM 2000). For example, within linear algebra, Harel (1997) names "the ability to connect ideas" as an indicator of understanding a concept (p. 109). Because this paper focuses on how one student reasoned about solutions to $A \boldsymbol{x}=0$ or $A \boldsymbol{x}=\boldsymbol{b}$ to draw connections between other concept statements in the IMT, the remainder of this section highlights two veins of existing research: student reasoning regarding how the concepts in the IMT are related, and the notion of "solution".

\section{Concepts Within the Invertible Matrix Theorem}

The Linear Algebra Curriculum Study Group (Carlson et al. 1993) named a number of topics as necessary to be included in any syllabus for a first course in undergraduate linear algebra, such as matrix addition and multiplication, systems of linear equations, properties of $\mathbb{R}^{n}$, and eigenvectors and eigenvalues. These concepts and others are the very ones linked together in the IMT. Furthermore, the IMT marries mathematical concepts from a variety of contexts; some concept statements in the IMT are vectororiented (such as span and linear independence), whereas others relate to linear transformations (such as one-to-one and onto). Equivalence and the importance of $A$ being an $n \times n$ matrix are also contributing factors as students develop ways of reasoning about connections among the various concept statements.

Although many studies address more than one of the key concepts, few explicitly investigate how students build relationships between the ideas as a main object of inquiry; a handful of studies address students' connections explicitly. For instance, Stewart and Thomas (2008) studied the understandings of linear independence and span that students need to construct an understanding of basis. The study participants were asked to create concept maps linking the notions of span, linear combination, basis, linear independence, and subspace. Some created concept maps that connected basis with span and linear independence, and some were missing one or both of these links. By comparing these concepts maps to a genetic decomposition of basis, the authors were able to posit potential pedagogical changes, such as an increased focus on the notion of linear combination or development of a more "embodied view" of basis.

Bogomolny (2007) investigated how example-generation tasks can elucidate student understanding of linear algebra. Bogomolny asked students to generate an example of a $3 \times 3$ matrix $A$ with nonzero entries such that the column vectors of $A$ formed a linearly dependent set. She found that many students utilized the connections "linear dependence $\leftrightarrow$ free variables / pivot positions / zero row in echelon form, and linear independence $\leftrightarrow$ no free variables / vectors not multiples of each other" (p. 271) to construct their examples. She hypothesized that the connections students relied upon were indicators as to whether they had an action view of linear dependence (e.g., a matrix should be row-reduced and then examined for a zero row) or an object view of linear dependence (e.g., the vectors have the property that they are linear combinations 
of each other). Thus, Bogomolny contended that example-generations tasks have the potential to be effective assessment tools for instructors but also helpful learning tools for students.

\section{The Concept of Solutions in Linear Algebra}

The concept of "solution" is ubiquitous in mathematics, from grade school and continuing through university. What typifies a solution, however, varies across content areas. For instance, in high school algebra, determining a solution to a system of equations consists of determining what values satisfy the equations within the system. In differential equations, however, finding a solution to a system of differential equations may entail determining what functions satisfy the system of differential equation. Furthermore, in many cases such as modeling physical problems, solutions to differential equations cannot be expressed in closed form and must be solved numerically (Habre 2000). In linear algebra, a common difficulty for students is the change in symbolism (Harel 2000), which in turn complicates the notion of solution. From high school courses or single-variable calculus, students are accustomed to equations such as $c x=d$, where all three variables take their values from the real numbers. However, in the matrix equation $R \boldsymbol{x}=0, R$ is a matrix and both $\boldsymbol{x}$ and 0 are multi-component columns vectors. Finding the solution (if one exists) entails determining the values for each component of $\boldsymbol{x}$ such that the associated system of linear equations has solutions. Thus, even commonly used symbolism in linear algebra entails a new level of complexity for students.

Particular to the equation $A \boldsymbol{x}=\boldsymbol{b}$, Parker (2010) found that student weaknesses in understanding the concept of span may stem from an imprecise understanding of how to interpret the matrix equation $A \boldsymbol{x}=\boldsymbol{b}$. She found students thought of the $\boldsymbol{b}$ as the solution to the matrix equation, rather than the $\boldsymbol{x}$, and she posited that this interpretation "could follow from an everyday understanding of 'solution' as the conclusion or end result of something. Using this idea, the solution would appear at the end of an equation...rather than in the middle" (p. 249).

Based on research in student thinking, Larson and Zandieh (2013) lay out a framework of three prominent interpretations of the equation $A \boldsymbol{x}=\boldsymbol{b}$, highlighting the role of the vector $\boldsymbol{x}$ in each interpretation. The linear combination interpretation is when $\boldsymbol{b}$ is a linear combination of the column vectors of the matrix $A$, and $\boldsymbol{x}$ is the set of weights on the column vectors of $A$. The systems interpretation is when $\boldsymbol{x}$ is interpreted as the set of values that satisfy the system of equations corresponding to $A \boldsymbol{x}=\boldsymbol{b}$, and the transformation interpretation of $A \boldsymbol{x}=\boldsymbol{b}$ corresponds to a view in which an input vector $\boldsymbol{x}$ is transformed into the output vector $\boldsymbol{b}$ via multiplication by the matrix $A$ The utility of this framework is that it offers an analytic lens through which to make sense of student thinking via the variety of both correct and incorrect ways students blend and coordinate ideas. I return to this framework in the conclusion section.

In summary, the concept of solutions plays a central and important role throughout mathematics. In higher-level mathematics, student struggles include understanding what constitutes a solution, the complexity of utilizing and interpreting the symbolism involved, and navigating multiple interpretations for a solution of a given equation. This case study provides a compelling example of the productive and 
powerful ways of reasoning about solutions that are possible in introductory linear algebra.

\section{Analytical Framework}

Approaching genetic analysis through a foundation in anthropological research, Saxe (2002) and his colleagues (Saxe et al. 2009) analyze human development over time from three different strands as a way to account for some of the complex factors of development. The authors define microgenesis as the short-term process by which individuals construct meaningful representations in activity, ontogenesis as the shifts in patterns of thinking, and sociogenesis as the reproduction and alteration of representational forms that enable communication among participants in a community (Saxe et al. 2009). In this study, I utilized the strands of microgenetic and ontogenetic analysis, paired with Toulmin's Model of Argumentation (1969) to frame my investigation into Abraham's ways of reasoning about the IMT in isolation (microgenesis) and over time (ontogenesis).

The Toulmin model of argumentation (Toulmin 1969) is based upon a distinction between logical and substantial arguments. Toulmin claimed that arguments presented in discourse in some socio-historical context for a particular purpose (what he called substantial arguments), such as a justification for an already established claim in the court of law, often are structurally distinct from the formal, deductive structure of logical arguments thought to be inherent and absolute. In other words, some aspects of socially presented arguments are context dependent yet still function as acceptable justifications for particular claims. Toulmin described six main components of a substantial argument: claim, data, warrant, backing, qualifier, and rebuttal (see Fig. 2). The first three of these - claim, data, and warrant - are seen as the core of an argument. According to this scheme, the claim is the conclusion that is being justified, whereas the data is the evidence that demonstrates that claim's truth. The warrant is seen as the explanation of how the given data supports the claim, and the backing, if provided, demonstrates why the warrant has authority to support the data-claim pair.

This work has been adapted by many in the fields of mathematics and science education research (Krummheuer 1995; Rasmussen and Stephan 2008; Yackel 2001) as a tool to assess the quality or structure of a specific mathematical or scientific argument and to analyze students' evolving conceptions by documenting their argumentation

Fig. 2 Toulmin's model of argumentation

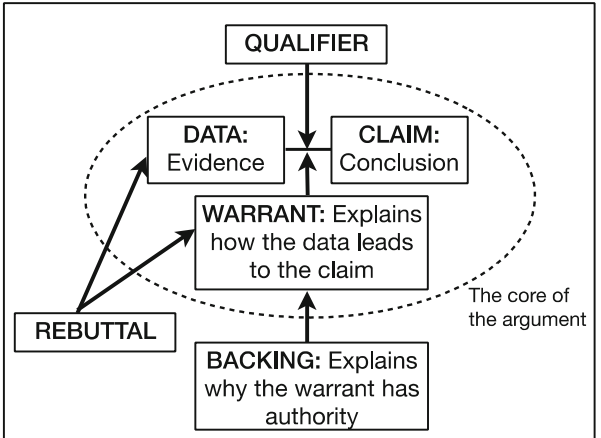


(Erduran et al. 2004; Inglis et al. 2007; Weber et al. 2008). In this paper, I use Toulmin's model to illuminate Abraham's argumentation as he reasoned about solutions to $A \boldsymbol{x}=0$ and $A \boldsymbol{x}=\boldsymbol{b}$. My use of "argumentation" is consistent with Krummheuer (1995) in that it relates to interactions that involve an intentional explication of the reasoning leading to a solution during or after its elaboration; argumentation is considered to be a "specific feature of social interaction" (p. 226). In this study, I analyzed Abraham's argumentation within whole class discussions, small group interactions, and individual interviews; each of these is considered a "social interaction". This is compatible with Aberdein's (2009) definition of argument as "an act of communication intended to lend support to a claim" (p. 1).

\section{Methods}

Data for this study came from the third iteration of a semester-long classroom teaching experiment (Cobb 2000) in an inquiry-oriented introductory linear algebra course. This work draws on the instructional design theory of Realistic Mathematics Education (RME) (Freudenthal 1991), which begins with the tenet that mathematics is a human activity. The intention was to create a linear algebra course that built on student concepts and reasoning as the starting point from which more complex and formal reasoning developed (details about the design research behind the course creation can be found in Wawro et al. 2013). The class analyzed comprised 30 students, including Abraham, and students enrolled had generally completed three semesters of calculus (at least two semesters were required). Approximately half had also completed a discrete mathematics course, most were in their second or third year of university, and student majors included computer engineering, computer science, mathematics, statistics, or another science or business field. Abraham was a junior statistics major who had completed three semesters of calculus and the discrete mathematics course. The class engaged in various RME-inspired instructional sequences focused on developing a deep understanding of key concepts such as span and linear independence (Wawro et al. 2012b), linear transformations (Wawro et al. 2012a), Eigen theory, and change of basis. These instructional sequences often involved engaging in a guided reinvention of key definitions or procedures. The textbook Linear Algebra and its Applications (Lay 2003) was used as a supplemental resource.

My rationale for choosing Abraham as the subject of my case study was two-fold. First, he was a member of a high-functioning small group in the linear algebra classroom. That is, Abraham and the members of his small group (a) with each other by pushing one another to explain their thinking and expecting participation from each member of the group; and (b) with the mathematics by staying on task, working through the given problems together, and displaying a genuine interest in exploring and understanding the content at hand. Second, Abraham was a high-functioning individual in the classroom community and his small group. That is, Abraham (a) was academically prepared in that he had successfully completed the requisite courses that positioned him for success in linear algebra; (b) was personally engaged and interested in the mathematics; and (c) was a key informant (Tremblay 1989) in that he possessed a unique ability to articulate his thinking and was willing to do so in whole class, small group, and interview settings. 
The data set for this study draws from the following four sources: video and transcripts of whole class discussion, video and transcripts of small group work, video and transcripts from individual interviews, and various written work. The whole-class and small group data came from the ten (out of 31) class sessions that were deemed most instrumental to the development of the IMT. To determine this, a day or portion of a day was deemed relevant for analysis if the classroom discourse either implicitly or explicitly involved the class members actively engaging in developing ways of reasoning about two or more concepts from the IMT in conjunction with each other. For each of those 10 days, I watched the video from Abraham's small group (which also captured work in whole class) and created a chronological summary of that class day's events. This included time-stamped information about the topic of discussion, the tasks or problems being investigated, and instances of small group work for that day. These descriptions indicated which sections of the video data were relevant for the research topics regarding the IMT, and thus became the small group work data for this study.

Abraham participated in two semi-structured individual interviews (Bernard 1988): one in the middle of the semester and one the week after final exams. The first interview was conducted before the IMT was fully developed in an explicit manner. Thus, this interview was composed mostly of questions that asked him to relate the ideas to which he had already been introduced (such as span and linear independence, but not determinants), to solve a given problem, or to generate examples or nonexamples. The post-semester interview explicitly asked him to reason about concept statements that comprise the IMT and how he saw them as equivalent. Finally, written work in the form of in-class worksheets, exams, reflections, homework, and portfolios, as well as Abraham's written work from the interviews, served as secondary data sources. The video for each of the 7 days of whole class discussion and small group work, and the video for both of Abraham's interviews, was transcribed completely by a professional transcriber; the relevant sections of the transcript were checked for accuracy and completeness.

To use Toulmin's Model as an analytical tool, I created an argumentation scheme for each of the claims found in the transcript. My determination of what in the transcript served as claim, data, warrant, backing followed the recommendation of Rasmussen and Stephan (2008) for documenting the structure and function of argumentation: claims "consist of either an answer to a problem or a mathematical statement for which the student may need to provide further clarification," data "usually involve the method or mathematical relationships that lead to the conclusion," warrants "are elaborations that connect or show the implications of the data to the conclusion," and backing serves to give validity to the core of the argument (p. 198). The authors comment that the function that a various utterance plays within an argument is critical to validly identify the various elements of the argument. That is, one utterance may serve as a claim that needs justified in one argument (e.g., the claim "any set of vectors containing the zero vector is linearly dependent") but serve as data in a different argument (e.g., the claim "the set $\left\{\left[\begin{array}{l}1 \\ 2\end{array}\right],\left[\begin{array}{l}0 \\ 0\end{array}\right]\right\}$ " is supported with data "any set of vectors containing the zero vector is a linearly dependent set").

For reliability purposes, at least one research colleague also constructed Toulmin schemes for a sample of arguments. As Rasmussen and Stephan (2008) state, this 
process is used to verify and/or refute each argument. Finally, each Toulmin scheme was given a unique label based on the day and order in which it occurred (e.g., Argument 20.1, Argument 20.2, Interview 2 Q6a Argument 4, etc.). The compilation of these created an argumentation $\log$ that identified each claim and supporting justification given. This process is consistent with Phase 1 of the methodology developed by Rasmussen and Stephan (2008) for documenting collective activity.

Ontogenetic analysis concerns shifts in patterns of thinking over time, and these shifts are documented by analyzing a learner's microgenetic processes at various stages of development. In this study, each individual Toulmin scheme is analogous to a microgenetic analysis regarding an explanation offered to serve the function of justifying a connection or solving a novel problem. The compilation and comparison over time of these Toulmin schemes is an ontogenetic analysis of Abraham's ways of reasoning about the IMT, revealing aspects such as what ideas in the IMT were used frequently and how the structure of his arguments changed over time. Thus, claims made about these arguments occur at two different levels. First, microgenetic analysis considers the structural layout as well as the mathematical content of each distinct argument that occurred at different moments throughout the semester. Ontogenetic analysis, by considering the collection of arguments and their relationship to one another, considers mathematical development, thus addressing how Abraham reasoned about the IMT over time.

\section{Results}

After coding Abraham's argumentation with Toulmin's Model, I noticed that reasoning about solutions to either $A \boldsymbol{x}=0$ or $A \boldsymbol{x}=\boldsymbol{b}$ was prominent in his argumentation about the IMT throughout the semester. Indeed, a search through the compilation of all Toulmin schemes of his argumentation revealed that the word "solution" appeared 103 times within the Toulmin schemes. From a search of ten key words within the coded transcripts (such as span, indepeden(t/ce), dependen(t/ce), reduce, vector, etc.), the word "solution" had a relative frequency of $21.2 \%$. This is in contrast to a relative frequency of $6.5 \%$ for "solution" in a search for the same ten keywords within whole class discussion transcripts. Furthermore, the occurrences of "solution" in Abraham's argumentation were distributed across the claim, data, warrant, and backing of the different Toulmin schemes in which it appeared.

There are two statements in the IMT developed in Abraham's class that explicitly use the word "solution" in their phrasing: "The only solution to $A \boldsymbol{x}=0$ is the trivial solution" and "For every $\boldsymbol{b}$ in $\mathbb{R}^{n}$, there exists a solution $\boldsymbol{x}$ to $A \boldsymbol{x}=$ $\boldsymbol{b}$ ". The results section highlights two themes in which Abraham used reasoning about solutions to either $A \boldsymbol{x}=0$ or $A \boldsymbol{x}=\boldsymbol{b}$ to justify connections between the other concept statements in the IMT. The first section is dedicated to an ontogenetic analysis of Abraham's justification of the equivalence of "The columns of $A$ form a linearly independent set" and "The columns of $A$ span $\mathbb{R}^{n}$." Additionally, Abraham reasoned about the notion of solution to make claims about nearly every other concept captured within the IMT: one-to-one and onto transformations, invertibility, determinants, null space, and eigenvalues. The second section of analysis addresses this way of reasoning with solutions. 


\section{Reasoning About Solutions to Form Connections Between Span and Linear Independence}

The two statements in the IMT developed in Abraham's class that explicitly use the word "solution" in their phrasing are: "The only solution to $A \boldsymbol{x}=0$ is the trivial solution" (listed as statement \#7 in the IMT, see Fig. 1), and "For every $\boldsymbol{b}$ in $\mathbb{R}^{n}$, there exists a solution $\boldsymbol{x}$ to $A \boldsymbol{x}=\boldsymbol{b}$ " (listed as statement \#3 in the IMT, see Fig. 1). The definition of linear independence developed in class was: "A set of vectors $\left\{\boldsymbol{v}_{1}, \boldsymbol{v}_{2}, \ldots, \boldsymbol{v}_{n}\right\}$ in $\mathbb{R}^{n}$ is a linearly independent set when the only solution to the equation $c_{1} \boldsymbol{v}_{1}+c_{2} \boldsymbol{v}_{2}+\ldots+\boldsymbol{c}_{\boldsymbol{n}} \boldsymbol{v}_{n}=0$ is if all $c_{1}, c_{2}, \ldots, c_{n}$ are zero". Once matrix equations were introduced, the class established that $A$ could be seen as an array of column vectors, and a matrix times a vector as the linear combination where the entries of $\boldsymbol{x}$ are the weights for the respective columns of $A$. Thus, by definition, statement \#7 in the IMT is equivalent to "The columns of $A$ form a linearly independent set" (statement \#6 in the IMT, see Fig. 1). Further note this equivalence holds true for any $m \times n$ matrix. Regarding the other statement in the IMT that uses the word "solution" (statement \#3), the class had developed early in the semester a theorem (which had served as a foundation for the IMT) stating the equivalence of the first four statements in Fig. 1 for any $m \times n$ matrix

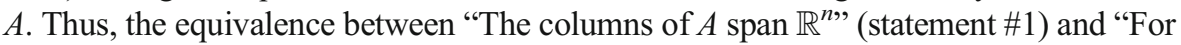
every $\boldsymbol{b}$ in $\mathbb{R}^{n}$, there exists a solution $\boldsymbol{x}$ to $A \boldsymbol{x}=\boldsymbol{b}$ " (statement \#3) was established in the classroom community.

When $A$ is an $n \times n$ matrix, as is the case in the IMT, not only do the two aforementioned equivalencies hold true (between \#3 and \#7, and between \#6 and $\# 1$ ), all four of the statements become equivalent. Of interest in this section is analyzing how Abraham reasoned about the equivalence of span and linear independence. Specifically, I use Toulmin's scheme to perform multiple microgenetic analyses of

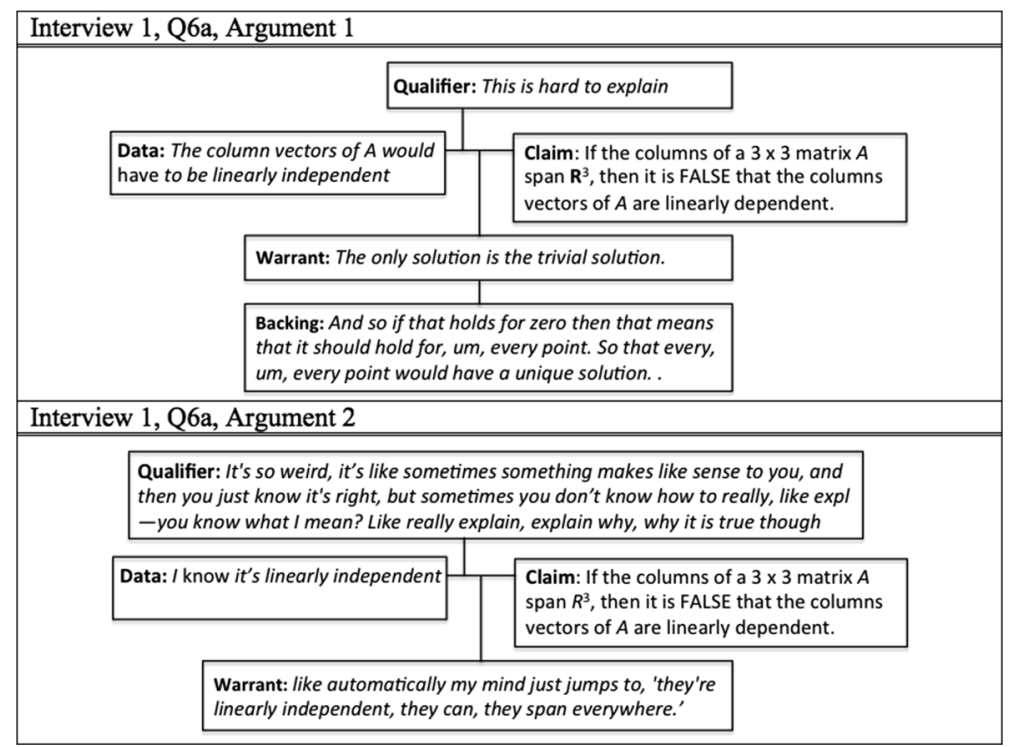

Fig. 3 Abraham reasons that if the columns of a $3 \times 3$ matrix $A$ span $\mathbb{R}^{3}$, then it is FALSE that the columns vectors of $A$ are linearly dependent 
Abraham's reasoning about linear independence and span, as well as to facilitate an ontogenetic analysis of the shifts in Abraham's reasoning over time. The arguments I analyzed here were chosen because of the central role reasoning about solutions (to either $A \boldsymbol{x}=0, A \boldsymbol{x}=\boldsymbol{b}$, or both) held in Abraham's discourse.

During both of the individual interviews (one conducted midway and one conducted at the end of the semester), Abraham was asked to respond to the following prompt: "True or False: If the columns of a $3 \times 3$ matrix span $\mathbb{R}^{3}$, then the column vectors are linearly dependent." During both interviews, Abraham immediately responded correctly with "false". The following transcript provides Abraham's initial explanation of why he stated false ("A" stands for

Abraham, and "Int" stands for the interviewer) during Interview 1:

A: The column vectors of $A$, this is hard to explain, but the column vectors of $A$ would have to be linearly independent. Um... and that means, what does that mean exactly? I mean that means that the only solution is the, uh, trivial solution. I mean, well-

Int: What means that the only solution is the trivial solution?

A: That's interesting, I don't know if I thought about it like that, because usually the, that's the, trivial solution is for zero only, so how does that apply to any point? Oh! That would apply, usually in linearly independent definition, you know, then you have the only solution to, you know, the $c_{1} \mathbf{x}_{1}$ plus, you know, equals zero is $c_{1}=c_{2}=c_{3}=0$. [Int: Ok.] And so if that holds for zero, then that means that it should hold for, um, every point. So that every, um, every point would have a unique solution. [Int: Ok.] And um, like, uh, every point has a unique solution and it's linearly independent. I guess that just relates linear independence to not just zero but to all points, is a unique solution.

A: [Reads from the question prompt] And to 'explain your answer.' ... It's so weird, it's like sometimes something makes like sense to you, and then you just know it's right, but sometimes you don't know how to really, like expl-you know what I mean? Like really explain, explain, you know, why, why it is true though. I know it's linearly independent.

Int: Ok. You know that what's linearly independent?

A: Well I know that these 3 column vectors are linearly independent. But that like automatically my mind just jumps to, 'they're linearly independent, they can, they span everywhere'.

23 Int: Ok. Why does, why in your mind does 'they're linearly independent' mean that they span everywhere? And when you say 'everywhere,' what do you mean 'everywhere?'

25 A: I should be more specific. They, ok, they span all of $\mathbb{R}^{3}$.

26 Int: Ok. And you're talking again about the column vectors span all of $\mathbb{R}^{3}$ ? [A: Yes.]

This response is decomposed into two Toulmin schemes (see Fig. 3; Italics in Toulmin schemes indicate exact quotes throughout the manuscript). In this response, Abraham immediately claimed the statement was false and qualified his argument, stating it was "hard to explain" (line 1). The data he provided in an attempt to justify the claim was that "the column vectors of $A$ would have to be linearly independent" (lines 1-2), and his warrant was the definition of linear dependence (line 3). The interviewer asked for clarification of his justification, so Abraham supplied a backing that if $A \boldsymbol{x}=0$ had only the trivial solution, then there would be a unique solution to $A \boldsymbol{x}=\boldsymbol{b}$ for every $\boldsymbol{b}$ (lines 5-10). In this justification, Abraham seems confident the given implication is false, but he struggles to explain why. Mathematically, the explanation he provided argued that if the columns of a $3 \times 3$ matrix $A$ were linearly independent, then the 
column vectors of $A$ spanned $\mathbb{R}^{3}$; this is more closely aligned with the converse of the implication he was asked to verify or refute, rather than the implication itself. When Abraham explained his answer further, he reflected that sometimes you might know something is true but not know how to explain why (lines 14-18). Lines 20-26 then confirm that he was thinking in the direction "linear independence implies span".

Lines 1-26 and Fig. 3 provide the transcript and analysis of Abraham's initial response to the interview question; a deeper look into his response in Interview 1 reveals a pivotal moment in Abraham's reasoning. The remainder of his conversation with the interviewer, whose follow-up questions resulted in Abraham explaining why having a unique solution to $A \boldsymbol{x}=0$ would imply there is a unique solution for every $\boldsymbol{b}$ to $A \boldsymbol{x}=\boldsymbol{b}$, is given in lines $27-49$.

27 [17 s pause] I started to think about this, like when you have [writes $A \boldsymbol{x}=0$ ], this is $A \boldsymbol{x}=0$, that a lot of the

28 characteristics apply to $A \boldsymbol{x}=$ some vector $\boldsymbol{b}$ [writes $A \boldsymbol{x}=\boldsymbol{b}]$... But because, like, I have all these points here

29 [draws dots in an augmented matrix], ... and the 0's [writes zeroes in the rightmost column of the

30 matrix]... And then let's say I was, um, I had some other points [draws an empty augmented matrix with

31 last column $<1,2,3>$ ]. Ah, this, whatever I do over here with row reducing this [points to his original

32 augmented matrix], it's really only going to affect this [points to the coefficient matrix portion]. So

33 [repeats gesture on newer augmented matrix] if I row reduce both of these, no matter what they are [fills in

34 the dots on the new augmented matrix], like this side [taps paper repeatedly on the coefficient matrix side

35 of both augmented matrices] ends up being the same, you know, this part of it.

$38 \ldots$ If there's a unique solution, $c_{1}=0, c_{2}=0$ and $c_{3}=0$ for zero [points to the $\left.<0,0,0\right\rangle$ in the first augmented 39 matrix] then whatever, since, I mean, this [points back and forth between the two coefficient matrices]

40 when you row reduce it, it ends up being like the same thing on this side, then most of the time, there

41 should be a unique solution for any $\boldsymbol{b}$ over here [points to the $<1,2,3>$ in the second augmented matrix]. If,

42 if it like, I think of it translating, but there are cases where it doesn't, and I remember thinking of one,

43 where it doesn't work like that.

45 ... But there are cases where it doesn't, and I remember thinking of one, where it doesn't work like that. 46 And it's actually when the bottom row ends up being like that [writes " $0000 \mid 1$ "]. That's when there's [no 47 solution].

After clarifying remarks with the interviewer (lines 23-26), Abraham spontaneously stated, "I started to think about this, like when you have, this is $A \boldsymbol{x}=0$, that a lot of the characteristics apply to $A \boldsymbol{x}=$ some vector $\boldsymbol{b}$ " (lines 27-28). He then spent $5 \mathrm{~min}$ exploring, in what seemed to be a way new to him, how a relationship between solutions of these two equations would imply relationships between the concepts of span and linear independence (lines 29-47). This argument, whose Toulmin scheme is given in Fig. 4, was the first time Abraham clearly articulated that he was thinking about a relationship between solutions to $A \boldsymbol{x}=0$ and $A \boldsymbol{x}=\boldsymbol{b}$.

Although the claim "when you have, this is $A \boldsymbol{x}=0$, that a lot of the characteristics apply to $A \boldsymbol{x}=$ some vector $\boldsymbol{b}$ " (lines 27-28) is somewhat vague, his data and warrant serve to provide support for this claim by arguing that one characteristic that "applies" is that of unique solutions. Within his data, Abraham stated that when both systems are converted to augmented matrices, the coefficient matrix is identical in both. Thus, if you follow the same row-reducing steps, the final row-reduced echelon form of $A$ in $A \boldsymbol{x}=\boldsymbol{b}$ will be the same as that in $A \boldsymbol{x}=0$ (lines 28-34). In his warrant, he stated a unique solution to $A \boldsymbol{x}=0$ would imply that "most of the time, there should be a unique solution 
for any $\boldsymbol{b}$ " in his $A \boldsymbol{x}=\boldsymbol{b}$ system (lines $38-42$ ). The only elaboration provided for that somewhat unclear warrant was his qualifier, wherein he stated he remembered that if the bottom row of a row-reduced augmented matrix looks like [ $\left.\begin{array}{llll}0 & 0 & 0 & 0\end{array}\right]$, that there would be no solution to $A \boldsymbol{x}=\boldsymbol{b}$ (lines 45-47). It could be that Abraham was recalling a situation related to a non-square matrix $A$ (namely, if $A$ is an $n \times m$ matrix with $n<m$, then it is possible to have a unique solution to $A \boldsymbol{x}=0$ but no solution to $A \boldsymbol{x}=\boldsymbol{b}$ for some $\boldsymbol{b} \in \mathbb{R}^{\boldsymbol{m}}$ ); however, [ [ $\left.\begin{array}{lll|l}0 & 0 & 0 & 1\end{array}\right]$ could not occur when there exists a unique solution to $A \boldsymbol{x}=0$ for an $n \times n$ (square) matrix $A$, and Abraham failed to recognize that.

The previous example of Abraham reasoning about $A \boldsymbol{x}=0$ and $A \boldsymbol{x}=\boldsymbol{b}$ together stemmed from a question which asked him to reason about whether or not three vectors spanning $\mathbb{R}^{3}$ implied the vectors were linearly dependent. Although he knew that this implication was false (see Fig. 3), how to reason about that implication by reasoning about solutions (or unique solutions) was a developing idea for him. Furthermore, he reasoned about what the zero vector being the only solution to $A \boldsymbol{x}=0$ implied when reasoning about the solutions to $A \boldsymbol{x}=\boldsymbol{b}$ for all possible $\boldsymbol{b}$. Given that this line of inquiry was not one that surfaced cleanly during whole class discussion, its originality with Abraham is worth noting.

The next argument under consideration occurred during whole class discussion on Day 31, when Abraham mentioned unique solutions in conjunction with both span and linear independence. The class was presenting work about which concept statements from the IMT were "most obviously equivalent" for their small group, and Justin, a member of Abraham's small group, had just presented an explanation for why his group thought the following three statements are most readily seen as equivalent: (a)

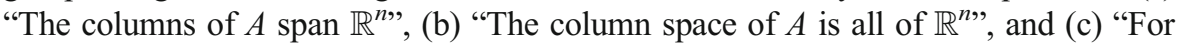
every $\boldsymbol{b}$ in $\mathbb{R}^{n}$ there is a way to write $\boldsymbol{b}$ as a linear combination of the columns of $A$." Abraham was preparing to explain why a fourth statement, "For every $\boldsymbol{b}$ in $\mathbb{R}^{n}$ there exists a solution $\boldsymbol{x}$ to $A \boldsymbol{x}=\boldsymbol{b}$," could join that category when Justin tried to add in a fifth, "the only solution to $A \boldsymbol{x}=0$ is the trivial solution." Abraham, however, was quick to respond that, for him, that fifth statement did not automatically belong in the category with the other four. The Toulmin scheme for his response is given in Fig. 5.

Abraham claimed that, for him, the fifth statement only belonged in the category if he were to add the word "unique" to the statement, "For every $\boldsymbol{b}$ in $\mathbb{R}^{n}$ there exists a solution $\boldsymbol{x}$ to $A \boldsymbol{x}=\boldsymbol{b}$ ". His data was that adding the word "unique," so that the statement read, "For every $\boldsymbol{b}$ in $\mathbb{R}^{n}$ there exists a unique solution $\boldsymbol{x}$ to $A \boldsymbol{x}=\boldsymbol{b}$," would imply that the column vectors of $A$ are linearly independent. He finished by saying, "but without the word [unique], I just think of "we can get to every $\boldsymbol{b}$." He did not provide a rationale for why adding the word "unique" to the statement would imply the column vectors were linearly independent, and he was not asked for justification. Argument 31.6 demonstrates a shift in Abraham's ability - from the first interview midway in the semester to the last day of class - to reason about these concepts in conjunction with one another. During the first interview (see Figs. 3 and 4), Abraham struggled to explain how he understood the connection between linear independence and span when $A$ is $n \times n$. He was unable to provide data or warrants to adequately support his claims, and he made claims regarding how solutions to $A \boldsymbol{x}=\boldsymbol{b}$ or $A \boldsymbol{x}=0$ related to each other or to span and linear independence about which he was uncertain and partially incorrect. On Day 31 , he seems more confident in his understanding of the concepts' relationship and distinction, 


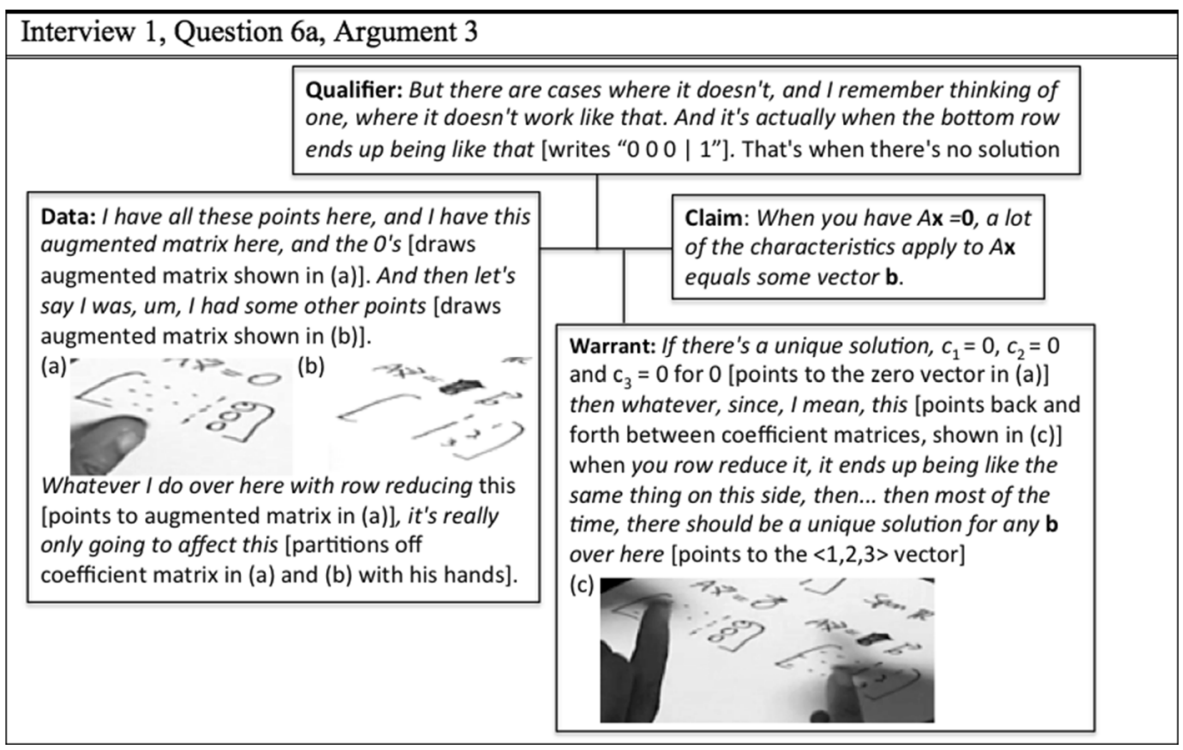

Fig. 4 Abraham reasons that some characteristics of $A \boldsymbol{x}=0$ apply to $A \boldsymbol{x}=\boldsymbol{b}$

as evidence in his decisive and quick attention to Justin's suggestion. Abraham's data relied on how uniqueness of solutions to $A \boldsymbol{x}=\boldsymbol{b}$ implied linear independence. Here Abraham used as data that which he had previously needed to justify. This ontogenetic analysis of shift in form and function of Abraham's argumentation, facilitated by the use of Toulmin's Model, shows a transition in Abraham's ways of reasoning about solutions to $A \boldsymbol{x}=\boldsymbol{b}$ or $A \boldsymbol{x}=0$, from one of uncertainty to one of great ease and utility.

The final example of Abraham reasoning about solutions to $A \boldsymbol{x}=0$ and $A \boldsymbol{x}=\boldsymbol{b}$ to form connections between span and linear independence is Argument 1 from Interview 2, Question 1a. In this argument, Abraham was responding to the prompt, "True or False: If the columns of a $3 \times 3$ matrix span $\mathbb{R}^{3}$, then the column vectors are linearly dependent". Note this is the same prompt he was responding to in the arguments presenting in Figs. 3 and 4 but with 2 months having passed between his two responses.

1 That would be false. Let's see. [7-s pause] So if, the way I think of it is, if it, if it's spanning

2 a $3 \times 3$ matrix [draws the outer brackets for a matrix], um, then it's going to have like, you

3 know, 3 pivot positions here [writes 3 ones in on the diagonal]. And for like a square

4 matrix, I just think like if this is 3 pivots in each row, then it's also going to be,

5 automatically going to be 3 pivots in each column. And that way you're always going to

6 have a linearly independent set of...of, um, like $x$ equals something, $y$ equals something, $z$

7 equals something, because of that. And then that's, so that's going to be, basically a unique

8 solution for every output. So let's see, so [writes $A \boldsymbol{x}=\boldsymbol{b}$ ], for every $\boldsymbol{b}$ [taps the $\boldsymbol{b}$ ] there's a

9 unique $\boldsymbol{x}$ vector [taps the $\boldsymbol{x}$ ], which is [writes a vector $<x, y, z>$ next to the matrix] I guess

10 if you want to think of, I don't know, this vector [taps on the vector that he wrote next to

11 the matrix then writes an equals sign], this output $b$ [draws two brackets after the equals

12 sign]. There's a unique $\langle x, y, z>$ vector such that $A \boldsymbol{x}=\boldsymbol{b}$ in the output, so that'd be linearly

13 independent, but not dependent.

As he had done in Interview 1, Abraham claimed the statement was false. The structure of his justification in Interview 2, however, shifted. First, he used as data that 
$A$ had three pivots, which led to a warrant that for "a square matrix, I just think like if this is three pivots in each row, then it's also going to be, automatically going to be three pivots in each column". Finally, for a backing, he stated, "so that's going to be, basically a unique solution for every output... so that'd be linearly independent, but not dependent". In the latter portion, there appeared to be an implicit assumption that a unique solution for $A \boldsymbol{x}=\boldsymbol{b}$ for every $\boldsymbol{b}$ implies linear independence (this would be valid, for the zero vector would be such a vector $\boldsymbol{b}$ and $A 0=0$ for any matrix $A$ because it defines a linear transformation). Abraham's previous argumentation schemes support his eventual ability to reason in this way, or, even more so, to reason about, "there exists a unique solution to $A \boldsymbol{x}=\boldsymbol{b}$ for every $\boldsymbol{b}$," as an alternative definition of linear independence.

To aid in comparing the two responses, Table 1 has the Toulmin schemes of his response from the Interview 1 (Fig. 3) in the leftmost column and the Toulmin schemes of his response from Interview 2 in rightmost column. Here I draw attention to the two levels of backing within this argument. Recall that during Interview 1, Abraham had difficulty explaining why the correct answer to the prompt's question was "false," with his first two arguments basically conveying that he "just knew" it was so. His third argument in that Interview 1 response indicated he was beginning to think in terms of unique solutions (Fig. 4). As shown in the right side of Table 1, however, he used this very thing that he struggled with in Interview 1 as backing for his claim during Interview 2. In other words, he justified that a spanning set of vectors were linearly independent by reasoning about unique solutions to $A \boldsymbol{x}=\boldsymbol{b}$. Through ontogenetic analysis focused on a comparison of Interview 1 and 2, a dramatic shift in Abraham's ways of reasoning is illuminated. Furthermore, nuances of this evolution of reasoning are shown plausible when additional arguments (namely those presented in Figs. 4, 5, 6, and 7) are considered.

\section{Reasoning About Solutions to Support Claims About Other Concepts}

Abraham also used solutions to reason about relationships between other concept statements within the IMT. Examples for each relevant concept are: free variables (during Interview 1); one-to-one (during whole class discussion and Interview 2); onto (during Interview 2); determinants (during Interview 2); null space (during whole class discussion and Interview 2); and eigenvalues (during Interview 2). I conclude by detailing two of these as exemplars.

Consistent with the argument from Day 31 given in Fig. 5, consider the following explanation from Interview 2, which occurred about a week after Day 31. During Interview 2, Abraham was asked to arrange the 16 statements from the IMT (which

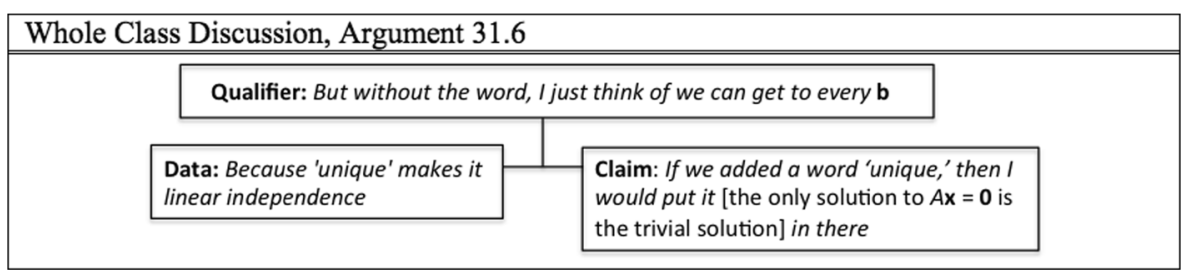

Fig. 5 Abraham explains why the 'trivial solution' does not belong with the other statements 
Table 1 Toulmin schemes for Abraham's two interview responses to the prompt regarding an implication between span and linear dependence

Prompt: Suppose you have a 3 by 3 matrix $A$ and you know that the columns of $A$ span $\mathbb{R}^{3}$. Decide if the following statement is true or false, and explain your answer: The column vectors of $A$ are linearly dependent.

\section{Int 1 Q6a Arg 1}

Claim: If the columns of a $3 \times 3$

matrix $A$ span $\mathbb{R}^{3}$, then it is FALSE

that the columns vectors of $A$ are linearly dependent.

Data: The column vectors of $A$ would have to be linearly independent

Qualifier: This is hard to explain

Warrant: The only solution is the trivial solution (Interviewer asks for clarification of the warrant)

Backing: And so if that holds for zero then that means that it should hold for, um, every point. So that every, um, every point would have a unique solution.

(Reads "explain your answer" prompt and responds)

Int 1 Q6a Arg 2

Claim: If the columns of a $3 \times 3$ matrix $A$ span $\mathbb{R}^{3}$, then it is FALSE that the columns vectors of $A$ are linearly dependent.

Data: I know it's linearly independent

Qualifier: It's so weird, it's like sometimes something makes like sense to you, and then you just know it's right, but sometimes you don't know how to really, like expl-you know what I mean? Like really explain, explain why, why it is true though.

Warrant: like automatically my mind just jumps to, 'they're linearly independent, they can, they span everywhere.'

\section{Int 2 Q1a Arg 1}

Claim: If the columns of a $3 \times 3$ matrix $A$ span $\mathbb{R}^{3}$, then it is FALSE that the columns vectors of $A$ are linearly dependent.

Data: Matrices whose columns span $\mathbb{R}^{3}$ have three pivot positions

Warrant: And for like a square matrix, I just think like if this is three pivots in each row, then it's also going to be, automatically going to be three pivots in each column.

Backing: And that way you're always going to have a linearly independent set of...of, um, like $x$ equals something, y equals something, $z$ equals something, because of that.

Backing2: And then that's, so that's going to be, basically a unique solution for every output...There's a unique $<x, y, z>$ vector such that $A \mathbf{x}=\mathbf{b}$ in the output, so that'd be linearly independent, but not dependent.

were printed on individual cards) into piles that, for him, "went together". As he and the interviewer discussed his choices, the interviewer asked Abraham to discuss a grouping he had not originally made: "I was wondering if you can pull off [to the side] 'one-toone,' 'onto' and ' $A$ is invertible'. And can you talk about, do you have any way to connect these three ideas together?" Abraham's response culminated in a claim that "one-to-one and onto together" implies there is a unique solution to $A \boldsymbol{x}=\boldsymbol{b}$; he did not address invertibility in his response. His written response is given in Fig. 6. For brevity, the full transcript and Toulmin analysis of his initial response is omitted.

First, note that Abraham gave non-standard definitions for onto and one-to-one transformations (see Fig. 6): for "onto," he wrote, "for every $\vec{b}$ there is at least one $\vec{x}$ such that $A \vec{x}=\vec{b}$. For one-to-one, as he wrote, he stated, "one-to-one, I'm going to say at most. Let's see if I can say it exactly the same way. For every $\vec{b}$ there's at most one $\vec{x}$ such that $A \vec{x}=\vec{b}$ ". These formulations are nonstandard because one-to-one and onto are defined as characteristics of functions (the definitions given in class are: 


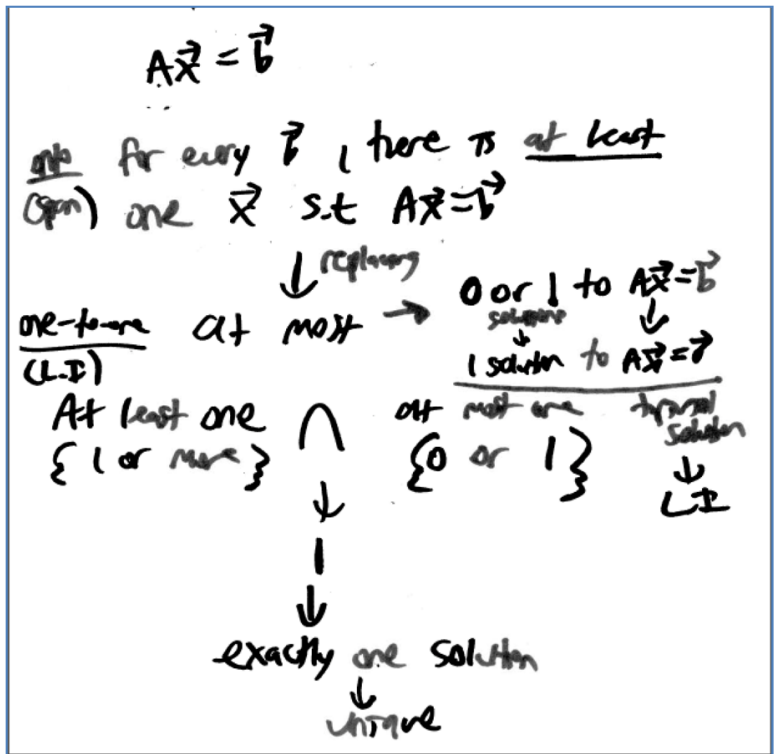

Fig. 6 Abraham's written response to how he connects the ideas of "one-to-one," "onto," and " $A$ is invertible" together

Let $T: \mathbb{R}^{n} \rightarrow \mathbb{R}^{m}$ be a transformation. $T$ is called one-to-one if for every $\boldsymbol{b} \in \mathbb{R}^{m}$ there exists at most one $\boldsymbol{x} \in \mathbb{R}^{n}$ such that $T(\boldsymbol{x})=\boldsymbol{b}$. $T$ is called onto $\mathbb{R}^{m}$ if for every $\boldsymbol{b} \in \mathbb{R}^{m}$ there exists at least one $\boldsymbol{x} \in \mathbb{R}^{n}$ such that $T(\boldsymbol{x})=\boldsymbol{b}$.), whereas Abraham defines them as if they are properties of matrix equations. Abraham's class only worked with $\mathbb{R}^{n}$ as vector spaces, and transformations $T: \mathbb{R}^{n} \rightarrow \mathbb{R}^{m}$ were shown to be able to be defined by a matrix representation $A$, where $T(x)=A \boldsymbol{x}$ for an $m \times n$ matrix $A$. As such, there are a couple of possible explanations for Abraham's definitions. Abraham may have been

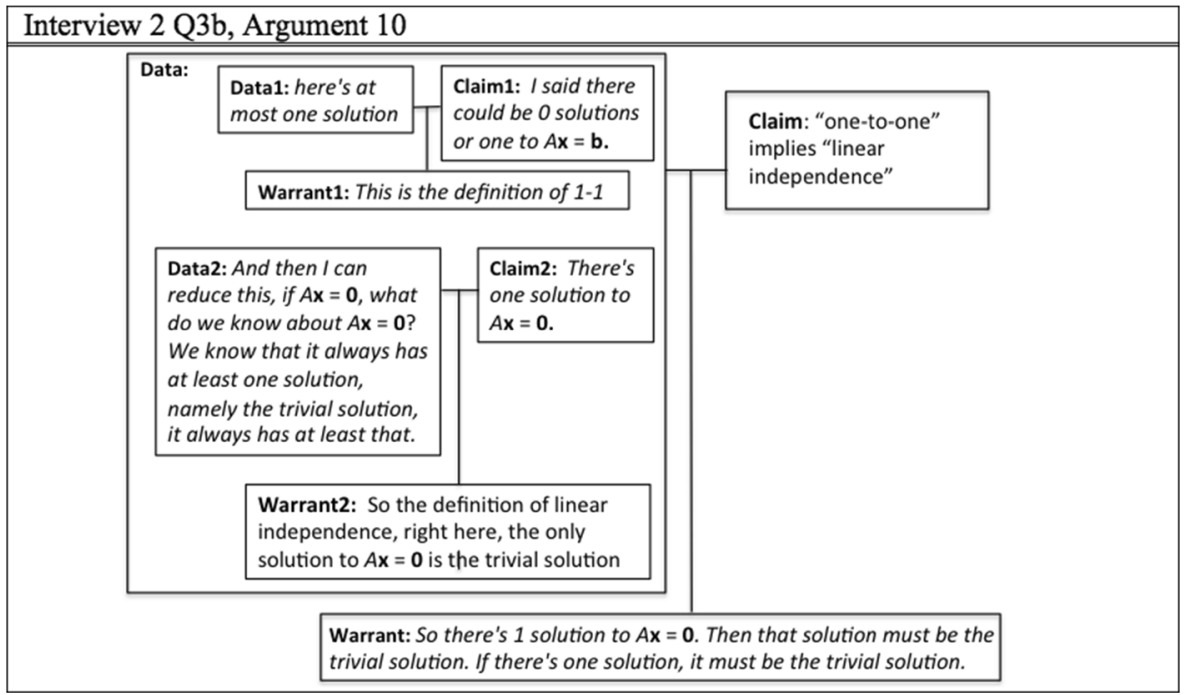

Fig. 7 Abraham explains how one-to-one and linear independence are related 
speaking metonymically (Lakoff and Johnson 1980) about the matrices as if they were themselves the linear transformations; alternatively, Abraham may have incorrectly assigned "one-to-one" and "onto" as properties of matrix equations.

Abraham argued that onto transformations have at least one solution and one-to-one transformations have either zero or one solutions. He wrote "span" under his definition of onto, pointed to his definition, and stated, "this is span". He wrote "L.I." (for linear independence) under his definition of one-to-one, pointed to this definition, and stated, "this is linear independence" (see Fig. 6). He noted the intersection of those possibilities implied that if a transformation is both one-to-one and onto, then for every $\boldsymbol{b}$ there would be exactly one solution $\boldsymbol{x}$ such that $A \boldsymbol{x}=\boldsymbol{b}$.

As a follow-up, Abraham was asked to further explain how one-to-one and linear independence were related for him (lines 2-3 in the below transcript); note the prompt is rather open-ended. Thus, Abraham could have discussed a relationship at a broad conceptual level, provided a proof of the concept statements' equivalence, or provided a proof of one direction of the implication. His response most closely resembles that the column vectors of the matrix associated with a one-to-one transformation would be linearly independent.

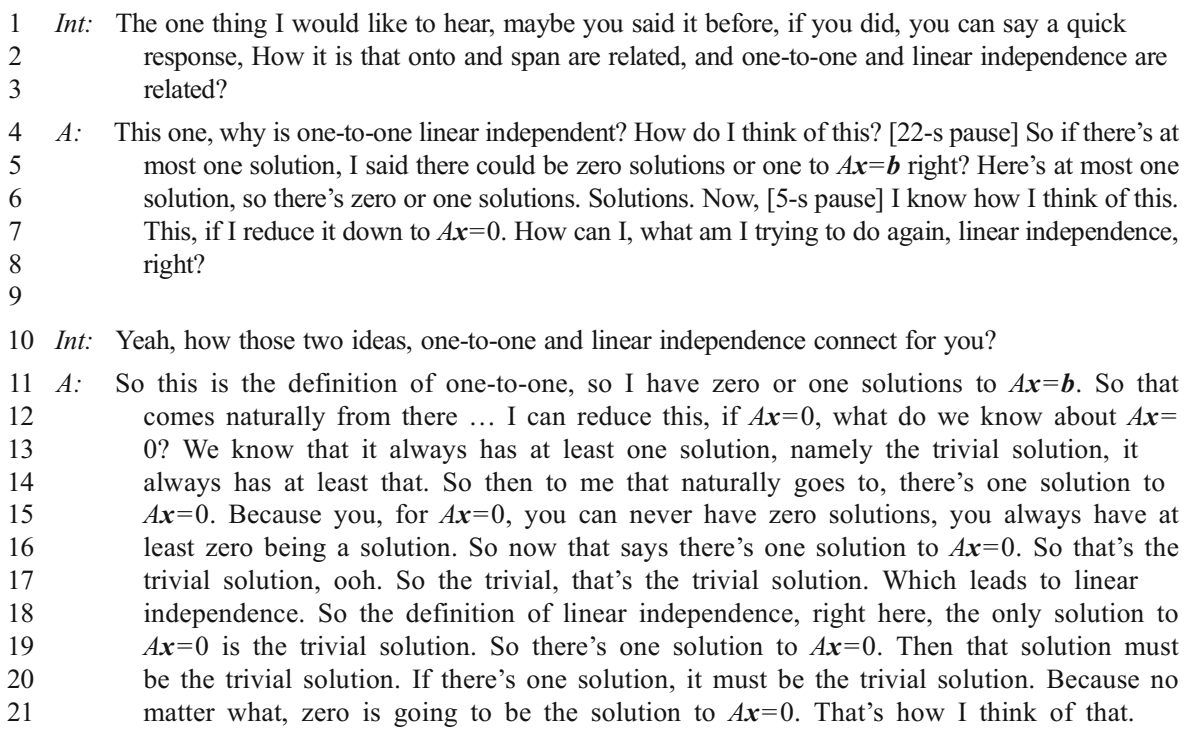
response, How it is that onto and span are related, and one-to-one and linear independence are related?

A: This one, why is one-to-one linear independent? How do I think of this? [22-s pause] So if there's at most one solution, I said there could be zero solutions or one to $A \boldsymbol{x}=\boldsymbol{b}$ right? Here's at most one solution, so there's zero or one solutions. Solutions. Now, [5-s pause] I know how I think of this. This, if I reduce it down to $A \boldsymbol{x}=0$. How can I, what am I trying to do again, linear independence, right?

10 Int: Yeah, how those two ideas, one-to-one and linear independence connect for you?

$11 A$ : So this is the definition of one-to-one, so I have zero or one solutions to $A \boldsymbol{x}=\boldsymbol{b}$. So that 12 comes naturally from there ... I can reduce this, if $A \boldsymbol{x}=0$, what do we know about $A \boldsymbol{x}=$ 0 ? We know that it always has at least one solution, namely the trivial solution, it always has at least that. So then to me that naturally goes to, there's one solution to $A \boldsymbol{x}=0$. Because you, for $A \boldsymbol{x}=0$, you can never have zero solutions, you always have at least zero being a solution. So now that says there's one solution to $A \boldsymbol{x}=0$. So that's the trivial solution, ooh. So the trivial, that's the trivial solution. Which leads to linear independence. So the definition of linear independence, right here, the only solution to $A \boldsymbol{x}=0$ is the trivial solution. So there's one solution to $A \boldsymbol{x}=0$. Then that solution must be the trivial solution. If there's one solution, it must be the trivial solution. Because no matter what, zero is going to be the solution to $A \boldsymbol{x}=0$. That's how I think of that.

During his response to the interviewer's question (line 1-3), Abraham said the word "solution" 21 times (lines 4-22), ten of which were noted in the Toulmin's scheme of this argument (see Fig. 7). In essence, Abraham responded by referring to the definitions of both one-to-one and linear independence, as well as the zero property of linear transformations (i.e., that $T(0)=0$ for any linear transformation $T$ ) to reason that if a given transformation is one-to-one, the column vectors of the associated matrix are linearly independent. Note that Abraham's discourse about one-to-one (that for every $\boldsymbol{b}$ there is at most one solution $\boldsymbol{x}$ such that $A \boldsymbol{x}=\boldsymbol{b}$ ) is consistent with his previous explanation of the concept. 
The Toulmin's Scheme for Abraham's response is composed of a claim, data comprising two sub-arguments, and a warrant. In the first sub-argument within the data, Abraham claims that based on the definition of one-to-one, there could be "zero solutions or one to $A \boldsymbol{x}=\boldsymbol{b}$ " (lines 5-6,11). Note that the data-warrant pair of this subargument previously served as a claim that needed justified for Abraham. Within the second sub-argument in Fig. 8, Abraham explained $A \boldsymbol{x}=0$ always has at least one solution, and if there is only one (the trivial solution), the column vectors of $A$ are linearly independent (lines 11-17). The warrant for the entire argumentation then concluded that if the only choice was for $A \boldsymbol{x}=0$ to have one solution, "it must be the trivial solution. Because no matter what, zero is going to be the solution to $A \boldsymbol{x}=0$. That's how I think of that" (lines 21-22). When reasoning about one-to-one transformations, Abraham relied on an interpretation of solutions to matrix equations (for every $\boldsymbol{b}$ there is at most one solution $\boldsymbol{x}$ such that $A \boldsymbol{x}=\boldsymbol{b}$ ) rather than linear transformations defined by a matrix representation. This may have allowed him to more easily deduce a connection to linear independence, which can also be defined in terms of solutions to the matrix equation $A \boldsymbol{x}=0$ (the only solution to $A \boldsymbol{x}=0$ is $\boldsymbol{x}=0$ ). Thus, this serves as an example of Abraham's sophisticated ways of reasoning about solutions to $A \boldsymbol{x}=0$ or $A \boldsymbol{x}=\boldsymbol{b}$ in a variety of situations.

The last example of Abraham reasoning about solutions to make connections between other concept statements in the IMT also comes from the second interview, as Abraham explained why he placed the cards "the number zero is not an eigenvalue of $A$ " and "the null space of $A$ contains only the zero vector" into a pile of concept statements that "went together" for him (this was part of the card sorting task previously

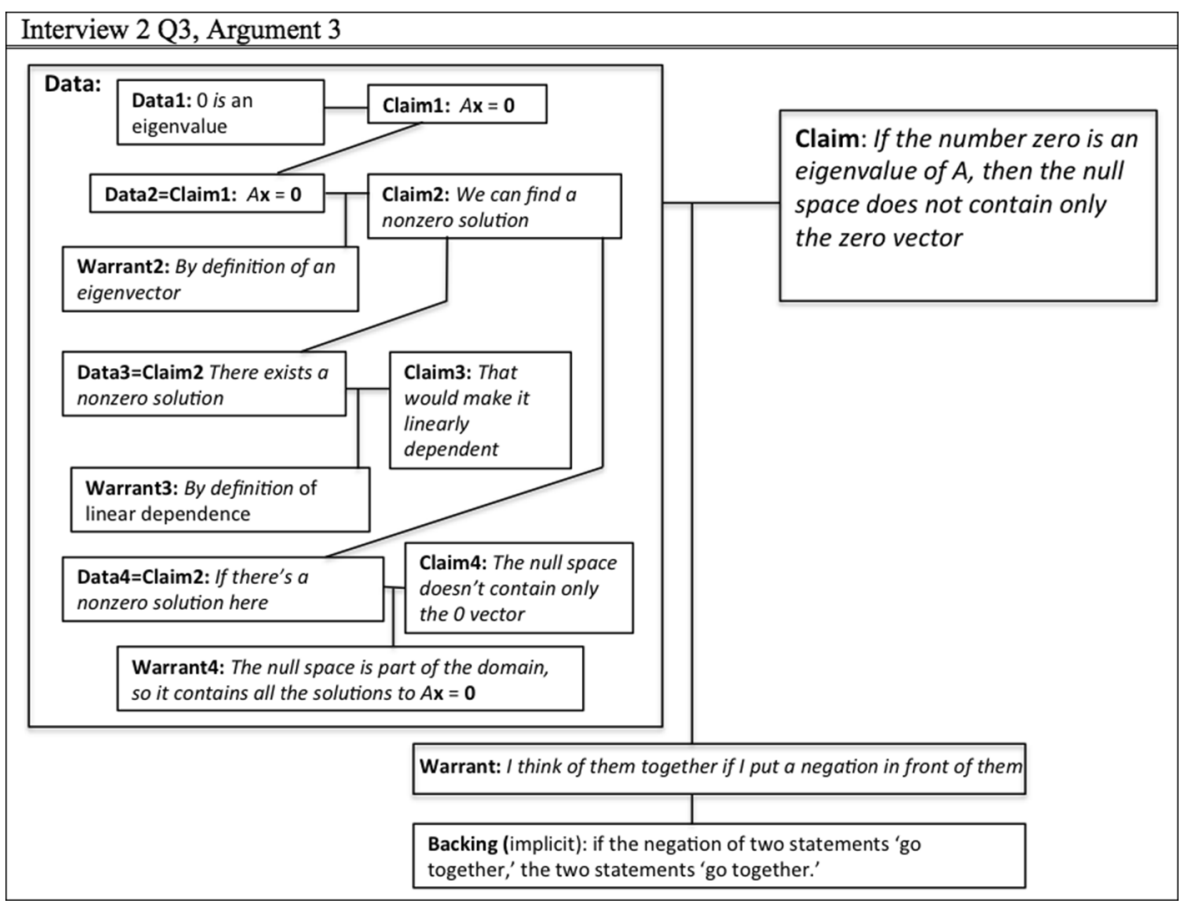

Fig. 8 Abraham explains how "zero is an eigenvalue" and " $N(A) \neq\{0\}$ " are related 
described). In a rather complex justification (which lasted nearly 10 min during the interview), Abraham justified that he relates the concept statements to each other by thinking about their negations. The Toulmin scheme for one direction of Abraham's argument, "If zero is not an eigenvalue of $A$ then the null space of $A$ does not contain only the zero vector" is given in Fig. 8.

When asked to connect the cards, "zero is not an eigenvalue of $A$ " and "the null space of $A$ is only the zero vector," Abraham first stated he liked to think of "zero is an eigenvalue of $A$ " instead. The remainder of his argument, thus, detailed why that negation implied the negation of the second statement, namely, "the null space of $A$ does not contain only the zero vector". The Toulmin scheme for his argument (see Fig. 8) is composed of complex data, a warrant, and an implicit backing. The structure of the data is an example of a sequential structure, which is a Toulmin scheme within which the data for one specific claim contains an embedded string of data-claim pairs, such that subclaims serve as data for subsequent claims (Wawro 2012).

Abraham began by stating, "I'm thinking of an eigenvalue's definition something's, those are nonzero $\boldsymbol{x}$ such that $A \boldsymbol{x}=\lambda \boldsymbol{x}$. And so if the number zero, $\ldots$ So then if it is, if $\lambda=0$ then $A \boldsymbol{x}=0$. And then by the definition of an eigenvector, we can find a nonzero solution". Here Abraham claimed that if zero is an eigenvalue of $A$ (Data1), then $A \boldsymbol{x}=0$ (Claim 1). Abraham repeated that claim as his next data for the claim that $A \boldsymbol{x}=0$ has a nonzero solution (Claim2). He supported why the data supported the claim by referencing the definition of eigenvector (Warrant2). He then repeated Claim 2 and used it as Data3 to support that having a nonzero solution implied the column vectors of $A$ were linearly dependent (Claim3); for Claim2 and Claim3, which relate to the existence of nonzero solutions, he provided warrants that referenced the definitions of eigenvalues and linear dependence, respectively. Abraham then paused, stating:

And how does this relate to null space for me? Because this is saying the number zero. Then I think of this because if there's a nonzero solution here, then the null space doesn't contain only the zero vector. So I think I think of them together, if I put a negation in front of them. Because then if the number zero is eigenvalue, then the null space of $A$ does not contain only the zero vector. The null space contains, the null space is part of the domain, so it contains all the solutions to $A \boldsymbol{x}=0$.

Abraham "backtracked" a bit, restating Claim2 as data for a new claim that the null space doesn't contain only the zero vector (Claim4). He provided a warrant for the Data4-Claim4 pair, that the null space is composed of all solutions to $A \boldsymbol{x}=0$, that gives information about how Data4 supports Claim4. Finally, Abraham concludes his argument with a warrant that he thinks of the two cards together via negation; this relies on an implicit backing that the negation of two statements go together then the two statements go together. Note, then, that this argument provides a justification for only one direction of the equivalence between "zero is not an eigenvalue of $A$ " and "the null space of $A$ contains only the zero vector".

Both of the main concepts within the argument in Fig. 8, those of eigenvalues of null space, were developed within the latter third of the semester for this classroom community. Furthermore, although the classroom community had established the inclusion of both of the statements, "the number zero is not an eigenvalue of $A$ " and "the null space of $A$ contains only the zero vector" in the IMT, they had never explicitly discussed how to justify the equivalence of these two statements in particular. Thus, Abraham's argument here is unique when compared to what was discussed in the 
classroom. Of further interest is that Abraham chose to associate those two concept statements with each other as ones that, for him, "went together". From considering Fig. 9, one can see how prominent and integral reasoning about solutions to $A \boldsymbol{x}=0$ (in particular, the existence of nontrivial solutions) was in how he justified the implication from zero as an eigenvalue to a nontrivial null space.

\section{Conclusion}

There is precedence within mathematics education research to characterize student understanding by the depth and flexibility by which one can recognize and make connections among ideas (Dorier and Sierpinska 2001; Harel 1997; NCTM 2000). In this paper, I contribute to this body of work by presenting results about the ways in which one student, Abraham, reasoned about solutions to $A \boldsymbol{x}=0$ and $A \boldsymbol{x}=\boldsymbol{b}$ to draw connections between other concept statements within the Invertible Matrix Theorem. The data were analyzed through the use of Toulmin's Model and coordinated with the microgenetic and ontogenetic strands of genetic analysis. This case study serves as an example of an individual student's prominence and success with reasoning about solutions in higher-level mathematics. Given the abundance of research literature that documents student difficulties in reasoning about solutions in mathematics, this serves as a contrasting example highlighting what deep understanding and complex justifications are possible for students as they engage in mathematics.

Abraham's mathematics serves to inform a broader theory towards how students reason about concept equivalence in linear algebra. The body of research details various modes of description (e.g., Hillel 2000) and interpretations (Larson and Zandieh 2013) that characterize the variety and diversity of students' conceptualizations in linear algebra. Abraham's reasoning about solutions - in various modes of description (e.g., "we can get to every $\boldsymbol{b}$ " in Fig. 5) embodies a travel description of solution) and in various interpretations (e.g., coordinating solutions to vector equations and matrix equations in Fig. 9) - lends insight into specific mechanisms by which students might reconcile meaning across representations of concepts in linear algebra.

For instance, consider Abraham's flexibility in symbolic representations and interpretations related to the solutions of the matrix equations $A \boldsymbol{x}=0$ and $A \boldsymbol{x}=\boldsymbol{b}$. I conjecture that Abraham's flexibility with various symbolic representations of the two matrix equations and how to interpret those afforded him ways to reason about what the existence and uniqueness of solutions to $A \boldsymbol{x}=0$ might imply about solutions to $A \boldsymbol{x}=\boldsymbol{b}$, and vice versa. For instance, Fig. 3 illustrates Abraham's reliance on the augmented matrix form of the two equations to reason about "when you have $A \boldsymbol{x}=0$, that a lot of

Fig. 9 Abraham writes two equivalent symbolic forms of $A \boldsymbol{c}=\boldsymbol{b}$

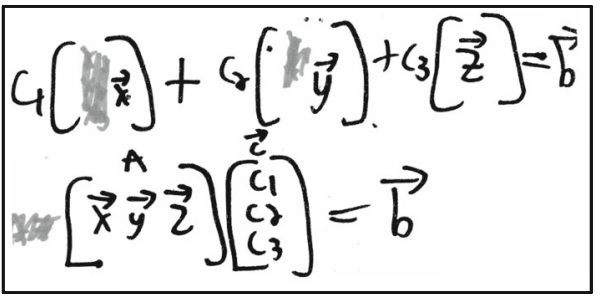


the characteristics apply to $A \boldsymbol{x}=\boldsymbol{b}$ ". First, he translated the given information from one symbolic representation (that of matrix equations) into another representation (that of augmented matrices). He then reasoned about row-reduction of the coefficient matrices in order to make claims about solutions to the augmented matrices, which he then translated back into matrix equations (see Fig. 3). While this argument was not completely accurate mathematically (he wasn't sure about when row reduction indicated nonexistence of solutions to a matrix equation), I contend the progress he made with reasoning about solutions in that argument was facilitated by his flexibility with symbolic representations.

Another example of his flexibility with symbolic representations occurred in Interview 1, shortly after the argument summarized in Fig. 3. Abraham was attempting

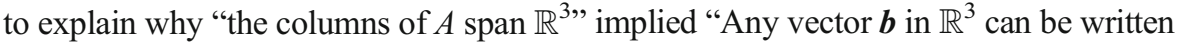
as a linear combination of the columns of $A$." Abraham stated, "I'm just rewriting it a different way to try to think about it," as he wrote the vector equation and corresponding matrix equation (see Fig. 9). The first representation in Fig. 9, the vector equation, corresponds to what Larson and Zandieh (2013) call the linear combination interpretation, in which the components of $\boldsymbol{x}$ are weights on the columns of $A$ that combine to produce the vector $\boldsymbol{b}$. A statement Abraham made in small group discussion a couple weeks prior in class, in which he said, "Because the weights are really what the solution is," supports the claim that Abraham could make this interpretation. Additionally, his statement during Interview 2 that "the null space is part of the domain, so it contains all the solutions to $A \boldsymbol{x}=0$ " is consistent with Larson and Zandieh's transformation interpretation of a matrix equation, in which $\boldsymbol{x}$ is an input vector that corresponds to an output vector 0 via transformation by the transformation defined by $A$. Further research needs to be conducted to investigate the plausibility of this conjecture, that Abraham's success with reasoning about solutions to $A \boldsymbol{x}=0$ and $A \boldsymbol{x}=\boldsymbol{b}$ was facilitated by his flexibility with various symbolic representations and interpretations of the two matrix equations.

Finally, this in-depth case study of Abraham's reasoning about solutions offers implications for future instruction in introductory linear algebra. Given that students often struggle with the abstract and proof-oriented nature of linear algebra, investigating one student's successful navigation provides insight into possible avenues to pursue in teaching such a course. Although not emphasized in this paper, the classroom community in which Abraham was a member was highly inquiry-oriented; that is, students were expected to both inquire into the mathematics in a genuine way, as well as be curious about and engage in other students' thinking about the content. On the other hand, the teacher facilitated this type of classroom environment by inquiring into student thinking and allowing that to help shape the direction of the classroom discourse. This type of environment may have played an integral role in fostering Abraham's ability to reason about the content and to verbalize his associated justifications clearly.

Second, a focused investigation into what ways of reasoning facilitated Abraham's success gives insight into what could be leveraged in the classroom for other students' benefit. For instance, Abraham was flexible in his use of symbolic representations, proficient in navigating the various interpretations of matrix equations, and explicit in referencing concept definitions within his justification (e.g., see Fig. 8). A linear algebra teacher may find it useful to purposefully emphasize these approaches within 
the classroom. This could be facilitated, for instance, by providing opportunities for students to reflect on the different symbolic forms and translations between them, as well as by having focused discussion on the importance of definitions within proof in mathematics. These implications would also be a profitable avenue for future research.

Acknowledgments This material is based upon work supported by the National Science Foundation under Grant Numbers DRL-0634074, DRL-0634099, and DUE-1245673. Any opinions, findings, and conclusions or recommendations expressed in this material are those of the authors and do not necessarily reflect the views of the National Science Foundation.

\section{References}

Aberdein, A. (2009). Mathematics and argumentation. Foundations of Science, 14(1-2), 1-8.

Bernard, R. H. (1988). Research methods in cultural anthropology. London: Sage.

Bogomolny, M. (2007). Raising students' understanding: Linear algebra. In J. H. Woo, H. C. Lew, K. S. Park, $\&$ D. Y. Seo (Eds.), Proceedings of the 31st conference of the international group for the psychology of mathematics education (Vol. 2, pp. 65-72). Seoul: PME.

Carlson, D. (1993). Teaching linear algebra: must the fog always roll in? The College Mathematics Journal, 24(1), 29-40.

Carlson, D., Johnson, C., Lay, D., \& Porter, A. D. (1993). The Linear Algebra Curriculum Study Group recommendations for the first course in linear algebra. The College Mathematics Journal, 24, 41-46.

Cobb, P. (2000). Conducting teaching experiments in collaboration with teachers. In A. E. Kelly \& R. A. Lesh (Eds.), Handbook of research design in mathematics and science education (pp. 307-330). Mahwah: Lawrence Erlbaum Associates.

Dorier, J.-L. (1995). Meta level in the teaching of unifying and generalizing concepts in mathematics. Educational Studies in Mathematics, 29, 175-197.

Dorier, J.-L., \& Sierpinska, A. (2001). Research into the teaching and learning of linear algebra. In D. Holton (Ed.), The teaching and learning of mathematics at university level: An ICMI study (pp. 255-273). Dordrecht: Kluwer Academic Publishers.

Dorier, J.-L., Robert, A., Robinet, J., \& Rogalski, M. (2000). The obstacle of formalism in linear algebra. In J.L. Dorier (Ed.), On the teaching of linear algebra (pp. 85-124). Dordrecht: Kluwer Academic Publisher.

Erduran, S., Simon, S., \& Osborne, J. (2004). TAPping into argumentation: developments in the application of Toulmin's argument pattern for studying science discourse. Science Education, 88(6), 915-933.

Freudenthal, H. (1991). Revisiting mathematics education. Dordrecht: Kluwer Academic Publishers.

Habre, S. (2000). Exploring students' strategies to solve ordinary differential equations in a reformed setting. The Journal of Mathematical Behavior, 18(4), 455-472.

Harel, G. (1989). Learning and teaching linear algebra: difficulties and an alternative approach to visualizing concepts and processes. Focus on Learning Problems in Mathematics, 11(2), 139-148.

Harel, G. (1997). Linear algebra curriculum study group recommendations: Moving beyond concept definition. In D. Carlson, C. R. Johnson, D. C. Lay, A. D. Porter, A. Watkins, \& W. Watkins (Eds.), Resources for teaching linear algebra (pp. 106-126). Washington: The Mathematical Association of America.

Harel, G. (2000). Three principles of learning and teaching mathematics: Particular reference to linear algebra - Old and new observations. In J.-L. Dorier (Ed.), On the teaching of linear algebra (pp. 177-190). Dordrecht: Kluwer Academic Publisher.

Hillel, J. (2000). Modes of description and the problem of representation in linear algebra. In J.-L. Dorier (Ed.), On the teaching of linear algebra (pp. 191-207). Dordrecht: Kluwer Academic Publisher.

Inglis, M., Mejia-Ramos, J. P., \& Simpson, A. (2007). Modelling mathematical argumentation: the importance of qualification. Educational Studies in Mathematics, 66, 3-21.

Krummheuer, G. (1995). The ethnology of argumentation. In P. Cobb \& H. Bauersfeld (Eds.), The emergence of mathematical meaning: Interaction in classroom cultures (pp. 229-269). Hillsdale: Erlbaum.

Lakoff, G., \& Johnson, M. (1980). Metaphors we live by. Chicago: The University of Chicago Press.

Larson, C., \& Zandieh, M. (2013). Three interpretations of the matrix equation $A \boldsymbol{x}=\boldsymbol{b}$. For the Learning of Mathematics, 33(2), 11-17.

Lay, D. C. (2003). Linear algebra and its applications (3rd ed.). Reading: Addison- Wesley. 
National Council of Teachers of Mathematics (2000). Principles and standards for school mathematics. Reston: Author.

Parker, C. F. (2010). How intuition and language use relate to students' understanding of span and linear independence in an elementary linear algebra class. Dissertation Abstracts International, 72(05). Retrieved July 30, 2012 from Dissertations \& Theses: Full Text. (Publication No. AAT 3448556).

Rasmussen, C., \& Stephan, M. (2008). A methodology for documenting collective activity. In A. E. Kelly, R. A. Lesh, \& J. Y. Baek (Eds.), Handbook of innovative design research in science, technology, engineering, mathematics (STEM) education (pp. 195-215). New York: Taylor and Francis.

Robert, A., \& Robinet, J. (1989). Quelques résultats sur l'apprentissage de l'algèbre linéare en première année de DEUG. Cahier de Didactique des Mathématiques, n53. Paris: IREM de Paris VII.

Saxe, G. B. (2002). Children's developing mathematics in collective practices: a framework for analysis. The Journal of the Learning Sciences, 11, 275-300.

Saxe, G., Gearhart, M., Shaughnessy, M., Earnest, D., Cremer, S., Sitabkhan, Y., et al. (2009). A methodological framework and empirical techniques for studying the travel of ideas in classroom communities. In B. Schwarz, T. Dreyfus, \& R. Hershkowitz (Eds.), Transformation of knowledge through classroom interaction (pp. 203-222). New York: Routledge.

Stewart, S., \& Thomas, M. O. J. (2008). Student learning of basis in linear algebra. In O. Figueras \& A. Sepúlveda (Eds.), Proceedings of 32nd PME conference, and XX PME-NA (Vol. 1, pp. 281-288). Morelia: PME.

Strang, G. (1988). Linear algebra and its applications (3rd ed.). San Diego: Harcourt Brace Jovanich.

Toulmin, S. (1969). The uses of argument. Cambridge: Cambridge University Press.

Tremblay, M. A. (1989). The key informant technique: A non-ethnographic application. In R. G. Burgess (Ed.), Field research: A sourcebook and manual (pp. 151-161). New York: Routledge.

Wawro, M. J. (2011). Individual and collective analyses of the genesis of student reasoning regarding the Invertible Matrix Theorem in linear algebra. (Doctoral dissertation). Retrieved from ProQuest Dissertations and Theses database. (Order No. 3466728).

Wawro, M. (2012). Expanding Toulmin's model: The development of four expanded argumentation schemes from analysis in linear algebra. In S. Brown, S. Larsen, K. Marrongelle, \& M. Oehrtman (Eds.), Proceedings of the 15th annual conference on research in undergraduate mathematics education (pp. 2-242-2-250). Portland, OR.

Wawro, M., Larson, C., Zandieh, M., \& Rasmussen, C. (2012). A hypothetical collective progression for conceptualizing matrices as linear transformations. In S. Brown, S. Larsen, K. Marrongelle, and M. Oehrtman (Eds.), Proceedings of the 15th Annual Conference on Research in Undergraduate Mathematics Education (pp. 1-465-1-479). Portland, OR.

Wawro, M., Rasmussen, C., Zandieh, M., Sweeney, G. F., \& Larson, C. (2012b). An inquiry-oriented approach to span and linear independence: the case of the magic carpet ride sequence. PRIMUS: Problems, Resources, and Issues in Mathematics Undergraduate Studies, 22(8), 577-599. doi:10.1080/ 10511970.2012.667516.

Wawro, M., Rasmussen, C., Zandieh, M., \& Larson, C. (2013). Design research within undergraduate mathematics education: An example from introductory linear algebra. In T. Plomp \& N. Nieveen (Eds.), Educational design research - part B: Illustrative cases (pp. 905-925). Enschede: SLO (Netherlands Institute for Curriculum Development).

Weber, K., Maher, C., Powell, A., \& Lee, H. S. (2008). Learning opportunities from group discussions: warrants become the objects of debate. Educational Studies in Mathematics, 68, 247-261.

Yackel, E. (2001). Explanation, justification and argumentation in mathematics classrooms. In M. van den Heuvel-Panhuizen (Ed.), Proceedings of the 25th international conference on the psychology of mathematics education (Vol. 1, pp. 9-23). Utrecht: PME. 